\title{
Geographical Prevalence of SARS-CoV-2 Variants, August 2020 to July 2021.
}

\author{
Wai Sing Chan \\ Hong Kong Sanatorium and Hospital \\ Yuk Man Lam \\ Hong Kong Sanatorium and Hospital \\ Janet Hei Yin Law \\ Hong Kong Sanatorium and Hospital \\ Tsun Leung Chan \\ Hong Kong Sanatorium and Hospital \\ Edmond Shiu Kwan Ma \\ Hong Kong Sanatorium and Hospital \\ Bone Siu Fai Tang ( $\nabla$ bsftang@gmail.com ) \\ Hong Kong Sanatorium \& Hospital
}

\section{Short report}

Keywords: COVID-19, SARS-CoV-2, variant, vaccine

Posted Date: September 14th, 2021

DOI: https://doi.org/10.21203/rs.3.rs-864626/v1

License: @ (1) This work is licensed under a Creative Commons Attribution 4.0 International License. Read Full License

Version of Record: A version of this preprint was published at Scientific Reports on March 18th, 2022. See the published version at https://doi.org/10.1038/s41598-022-08684-1. 


\section{Abstract}

Coronavirus disease 2019 (COVID-19) pandemic has progressed into second year. The etiologic agent severe acute respiratory syndrome coronavirus 2 (SARS-CoV-2) has evolved into numerous lineages, some of which are gifted with transmissibility advantages. This underscores the importance of variant surveillance, to keep public health countermeasures and our understanding on the virus up-to-date. In the light of this, we extracted one-year genomic data (August 2020 to July 2021) from Global Initiative on Sharing All Influenza Data (GISAID) EpiCoV ${ }^{\text {Tw }}$ database and estimated monthly proportions of 11 SARS-CoV-2 variants in various geographical regions. From continental perspective, delta variant of concern (VOC) predominated in Africa, Asia, Europe, North America and Oceania, with proportions ranging from 67.58 to $98.31 \%$ in July 2021. In South America, proportion of the expanding delta VOC (23.24\%) has been getting closer to the predominant yet diminishing gamma VOC (56.86\%). We further analyzed monthly data on new COVID-19 cases, new deaths, vaccination status and variant proportions of 6 countries. Similar to continental data, delta VOC predominated in all countries except Brazil in July 2021. In most occasions, rise and predominance of alpha, beta, gamma, delta and zeta variants were accompanied with surges of new cases, especially after the time points of major lineage interchange. The ascending phases of new cases lasted for 1 to 5 months with 1.69- to 40.63-fold peak growth, whereas new death toll varied with regional vaccination status. Our data suggested surges of COVID-19 cases might be predicted from variant surveillance data. Despite vaccine breakthroughs by delta VOC, death tolls were more stable in countries with better immunization coverage. Another takeaway is the urgent need to improve vaccine efficacy against delta and emerging variants.

\section{Background}

Lives evolve for adaptation and survival, and viruses are no exception. The nucleotide substitution rate of RNA viruses is high, typically ranges from $10^{-2}$ to $10^{-5}$ nucleotide substitutions per site, per year $(\mathrm{s} / \mathrm{n} / \mathrm{y})$ [1]. Severe acute respiratory syndrome coronavirus 2 (SARSCoV-2), the causative agent of coronavirus disease 2019 (COVID-19), is at the order of $10^{-4} \mathrm{~s} / \mathrm{h} / \mathrm{y}$ [2]. A wealth of mutations has accumulated as the pandemic progressed into second year $[3,4,5]$, shaping the genetic diversity of this virus over time, places and hosts.

SARS-CoV-2 is a single-stranded positive-sense RNA virus. Its genome is about 30,000-bp and comprises 11 genes. Among the gene products, spike protein plays a key role in host cell entry, the earliest essential step in viral life cycle [6, 7]. This outermost structural protein is thus a prime target of host immune system and under significant selection pressure, as evident by emergence of SARS-CoV- 2 variants.

As of 6 July 2021, World Health Organization (WHO) classified common SARS-CoV-2 variants into 4 variants of concern (VOCs), 4 variants of interest (VOIs) and 12 alerts for further monitoring, based on their clinical and public health impact [8]. The 4 VOCs are alpha (PANGO lineage B.1.1.7), beta (B.1.351/B.1.351.2/B.1.351.3), gamma (P.1/P.1.1/P.1.2) and delta (B.1.617.2/AY.1/AY.2) variants. The 4 VOls are eta (B.1.525), iota (B.1.526), kappa (B.1.617.1) and lambda (C.37) variants. The 12 alerts for further monitoring include 3 former VOls (epsilon, B.1.427/B.1.429; zeta, P.2 and theta, P.3), R.1/R.2, B.1.466.2, B.1.621, AV.1, B.1.1.318, B.1.1.519, AT.1, C.36.3/C.36.3.1 and B.1.214.2.

Continuous surveillance on genomic epidemiology of SARS-CoV-2 is essential to identify variants gifted with growth and transmissibility advantages, so as to keep public health countermeasures and our understanding on the virus up-to-date. The goal of this study was to appreciate recent geographical prevalence of SARS-CoV-2 variants.

\section{Methods}

\section{SARS-CoV-2 variants}

This study focused on 4 VOCs (alpha, beta, gamma and delta), 4 VOIs (eta, iota, kappa and lambda) and 3 former VOIs (epsilon, zeta and theta).

\section{Geographical prevalence of SARS-CoV-2 variants}

Geographical prevalence of selected variants was estimated from monthly proportions of whole genome sequences submitted to Global Initiative on Sharing All Influenza Data (GISAID) EpiCoV ${ }^{\mathrm{Tm}}$ database [9]. Data was extracted with following search criteria: complete genome ( $>29,000 \mathrm{bp}$ ), high coverage ( $<1 \%$ undefined bases), human host, collection date in 12 separate months (August 2020 to July 2021), location covering 6 continents (Africa, Asia, Europe, North America, Oceania and South America) and submission date up to 7 July 2021 for August 2020 to June 2021, and up to 8 August 2021 for July 2021.

\section{Further study on countries under the spotlight}


These included the USA, India, Brazil, the UK, South Africa and Israel. We looked for potential relationship between monthly data on variant proportions, new COVID-19 cases and deaths, and vaccination status in these countries. Variant prevalence data was extracted from GISAID EpiCoV ${ }^{T M}$ database as described above, and figures on new COVID-19 cases and deaths, and percentage of population vaccinated with at least 1 dose were extracted from Google News COVID-19 page [10].

\section{Results}

\section{Prevalence of SARS-CoV-2 variants in 6 continents}

Results are shown in Figure 1. Generally, all 6 continents encountered marked increase of total variant proportion between October and December. The variants expanded and their proportions were over $50 \%$ between December and March, and further rose to $85.77-100 \%$ in July.

The 6 continents displayed roughly 4 patterns of variant dynamics. In Africa, proportion of beta VOC fluctuated over the time and rose by about 5 folds from October to May. Proportion of alpha VOC (30.89\%) had climbed and approached that of beta VOC (31.69\%) in March, but then declined steadily to $0.41 \%$ in July. Delta VOC surpassed beta VOC in June and reached its prime at $71.27 \%$ in July.

In Asia, alpha VOC had been the major lineage from December and rose to the peak of $69.40 \%$ in May. It then started to diminish and was replaced by the rapidly expanding delta VOC in June, with the latter comprising $76.69 \%$ of submitted Asian genomes in July. Likewise, Europe and Oceania revealed similar dynamics with higher proportion of delta VOC in July ( $94.30 \%$ and $98.31 \%$, respectively).

In North America, epsilon variant was the most abundant from late 2020 to early 2021, with relatively low proportions of $5.71-16.42 \%$. It was later overridden by alpha VOC in March and the latter being taken over the place by delta VOC in July.

For South America, zeta variant outstood other variants from October (8.89\%) to January (37.51\%), followed by gamma VOC from February (41.93\%) to the peak of $85.29 \%$ in May, and dropped to $56.86 \%$ in July. Meanwhile, there was more than $20 \%$ rise of delta VOC in the same month.

\section{Monthly data on new COVID-19 cases, new deaths, vaccination status and variant prevalence of 6 countries}

Results are shown in Figure 2.

\section{The USA}

The USA has been leading in COVID-19 cases and deaths [10]. Her variant dynamics was very similar to that of North America, as $90 \%$ of North American genomes were collected in the USA. There were 2 ascending phases of new cases. The first one (October to December) was marked by peak growth of 5.27 folds accompanied with 3.34-fold increase in new deaths, which appeared to be less influenced by the 11 variants due to their low proportions (total variant proportion was $6.89 \%$ in December). In between 2 phases, alpha VOC overrode epsilon variant in March and had predominated up till June. Interestingly, new cases and deaths reduced gradually during this period, and vaccinated population rose to $55 \%$ by June. In the second ascending phase (July), new cases increased sharply by 3.84 folds whereas new deaths decreased by $16.43 \%$, with $58 \%$ of population vaccinated. Notably, it was accompanied with rapid expansion of delta VOC (+59\%) and big diminishment of alpha VOC (-44\%).

\section{India}

India has recorded second highest number of COVID-19 cases in the world, and ranked the third for death toll [10]. There were 2 ascending phases of new cases. The first one was accompanied with short and mild increase of new cases and deaths in September. COVID-19 vaccine was not available at that time, and total variant proportion was $0 \%$. The second one (March to May) was marked by large rebound of new cases and deaths (over 26-fold increase from baseline) in an under-vaccinated population (12\% by May), involving transition of 3 variants: kappa VOI diminished from the peak of $25.50 \%$ in March to $1.92 \%$ in May, with proportions of alpha and delta VOCs rising to $33.06 \%$ and $56.18 \%$ in the same month. In July, delta VOC expanded to $100 \%$ and $26 \%$ of Indian population was vaccinated, accompanied with decreasing trends of both new cases and deaths.

\section{Brazil}

Brazil has been ranked the third for COVID-19 cases and the second for death toll [10]. There were 3 ascending phases of new cases. The first one (November to January) involved about doubled peak growth of new cases and deaths, with $0.97 \%$ vaccination coverage by 
January. Zeta variant comprised the majority $(49.44 \%)$ and was approached by gamma VOC $(25.81 \%)$ in the same month. In the second phase (March), new cases and deaths increased by 1.69 and 2.19 folds, with $7.1 \%$ of population vaccinated. The diminishing zeta variant $(5.46 \%)$ was superseded by gamma VOC (83.96\%). In the third phase (July), new cases rebounded by about 2 folds and new deaths dropped by $31 \%$, with half of Brazilian population vaccinated. Meanwhile, gamma VOC has diminished to $62.78 \%$ and delta VOC has expanded to $23.07 \%$.

\section{The UK}

In the UK, there were 2 ascending phases of new cases. The first one lasted for 5 months (September to January), with 40.63- and 104.63fold increase of new cases and deaths in January. Alpha VOC comprised the majority of submitted genomes (83.94\%) and $14 \%$ of the UK population was vaccinated by the same month. The second one (May to July) was marked by 15.83- and 1.88-fold peak growth of new cases and deaths with 70\% vaccination coverage. Delta VOC has superseded alpha VOC and its proportion has got close to $100 \%$ in July.

\section{South Africa}

The number of COVID-19 cases in South Africa has been the highest in African continent [10]. There were 2 ascending phases of new cases. The first one lasted from October to January, with new cases and deaths increased by at least 6 folds. Vaccination coverage was $0 \%$, and beta VOC expanded to $93.89 \%$ in January. The second one has started from May to July, with 14.32 - and 7.56 -fold peak growth of new cases and deaths. By July, delta VOC (94.84\%) has overridden beta VOC (1.94\%), and only $10 \%$ of population was vaccinated.

\section{Israel}

Israel is one of the countries with leading vaccination rate [10]. There were 3 ascending phases of new cases. The first one was in September, with 2.89- and 1.67-fold increase in new cases and deaths. COVID-19 vaccine was not available at that time and variant proportion was $0 \%$. The second one (December to January) was accompanied with 9.64- and 4.73-fold peak growth of new cases and deaths. By January, alpha VOC predominated (56.17\%) and $34 \%$ of Israeli population was vaccinated. The third one (June to July) was marked by a sharp rise of new cases from low baseline (peak growth was 32.44 folds) and 10\% drop in new deaths. By July, delta VOC (96.68\%) surpassed alpha VOC (1.42\%) with 63\% vaccination coverage.

\section{Discussion}

COVID-19 pandemic has been the first of its kind caused by coronavirus. Since its outbreak in December 2019, it has led to 207,784,507 confirmed cases and 4,370,424 deaths worldwide (data accessed on 18 August 2021) [11]. There have been ups and downs of the situation, and in 2021 a number of countries have faced fresh COVID-19 resurgence, including but not limited to the 6 countries in this study. In addition to social factors like public health policy relaxation and pandemic fatigue, emergence of vaccine-escape variants was also an important contributing factor. Our data showed that in July, delta VOC has become the predominant lineage in Africa, Asia, Europe, North America and Oceania. In South America, its proportion has been increasing (23.24\%) and approached that of the predominant yet diminishing gamma VOC $(56.87 \%)$, which is not surprising as delta VOC was reported to be $40-60 \%$ more transmissible than alpha VOC [12].

From country-level temporal data, we might appreciate the expansion-diminishment patterns of various variants. For instance, the mean expansion-diminishment duration of alpha VOC was about 7.5 months, and the ascending and descending phases lasted for 4.75 and 2.75 months in average, respectively. In addition, another interesting observation was that the ascending phases of new cases were usually accompanied with rise and predominance of alpha (except the USA), beta, gamma, zeta, and most recently delta variants, or right after the time points of major lineage interchange. This might be (or might not be) accompanied with a rise in monthly new deaths, which appeared to be associated with immunization coverage. For instance, proportion of delta VOC in India rose sharply from March to April, surpassing alpha VOC and kappa VOI. In the meantime, both new cases and deaths increased drastically by 26.90 and 43.66 folds in May, with only $12 \%$ of Indian population vaccinated. Similar phenomenon was observed for the under-vaccinated population in South Africa. For the USA, the UK and Israel, more than half of their populations were vaccinated when delta VOC grew drastically to predominance. Their death tolls remained stable despite surges of new cases. Besides immunization coverage, availability of medical resources might also contribute to difference in mortality [13].

As of 1 June 2021, WHO validated 7 COVID-19 vaccines for emergency use, including 2 inactivated (Sinopharm and CoronaVac by Sinovac) and 5 RNA-based vaccines (BNT162b2 by Pfizer-BioNTech, AZD1222 by AstraZeneca (SK Bioscience, Serum Institute of India and European Union), and mRNA-1273 by Moderna) [14]. Emergence of SARS-CoV-2 variants has raised concern over vaccine efficacy 
against growing variety of lineages. A number of research groups reported vaccine-breakthrough cases involving SARS-CoV-2 variants and nearly all validated vaccines, for instance, gamma VOC against CoronaVac and AZD1222 in Brazil [15, 16]; 2 spike variants harbouring E484K and triple mutations (T95I, del142-144, and D614G) against mRNA-1273 and BNT162b2, respectively in the USA [17]; alpha and delta VOCs against AZD1222 and BNT162b2 in England [18, 19] and Scotland [20]; as well as delta VOC infecting an Everest trekker vaccinated with mRNA-1273 in Nepal [21]. Despite a broad range of cycle threshold values (15-33.3), the authors reported that all these vaccine-breakthrough cases involved reduced disease severity.

On the other hand, a number of studies focused on neutralizing activity of post-second-dose sera. For CoronaVac, Chen and coworkers reported that the neutralizing efficiency against pseudotyped lentiviruses of beta and gamma VOCs, and iota VOI were significantly reduced by factors of 5.27, 3.92 and 4.03, respectively, whereas efficiency against D614G, alpha VOC and epsilon variant were equally effective compared with the wildtype [22]. Their data also revealed that $5-34 \%$ of sera were capable of neutralizing the former 3 variants, compared to $82 \%$ against wildtype. Another study led by Cao and coworkers revealed that beta VOC pseudovirus or the authentic virus caused a major reduction in neutralization [23]. For BNT162b2, Lustig and coworkers revealed that neutralizing titers against alpha, delta and gamma VOCs were reduced by 1.7-2.6 folds, and 10.4 folds for beta VOC [24]. For AZD1222, Madhi and coworkers performed both pseudovirus and live virus assays [25]. They observed that $46 \%$ and $85 \%$ of post-second-dose sera lacked neutralization activity against pseudovirus with triple mutations (K417N, E484K and N501Y) and beta VOC pseudovirus, respectively. For live virus, $61.54 \%$ of postsecond-dose sera had undetectable neutralization activity against beta VOC, and the remainder with detectable activity were reduced by factors of 4.1-31.5. For mRNA-1273, Shen and coworkers reported that neutralization activity against epsilon variant and beta VOC pseudoviruses were 2-3 and 9-14 times lower than that against D614G pseudovirus, respectively [26].

Summing up the serological findings, delta VOC or other variants reduced neutralizing activity of vaccination-elicited antibodies at varying extents, with beta VOC being apparently more resistant. Beta VOC is characterized by 9 mutations in S1 subunit (D80A, D215G, 241del, 242del and 243del in N-terminal domain (NTD); K417N, E484K and N501Y in receptor-binding domain (RBD); as well as D614G) and a single mutation in S2 subunit (A701V near S1/S2 furin cleavage site) (Table 1). Among these signature amino acid changes, E484K and combination of K417N, E484K and N501Y might reduce the effectiveness of specific monoclonal antibody treatments [27]. K417, E484 and N501 are 3 of the 21 amino acids in RBD, the major target of neutralizing antibodies [28], which interact with human angiotensinconverting enzyme 2 (hACE2) [29]. Results of in vitro studies suggested E484K might play an indispensable role in escape of beta VOC from neutralizing antibodies [30] and loss of neutralizing activity of certain monoclonal antibodies [30,31,32]. Despite the difference in resistance against vaccine protection, beta and gamma VOCs share very similar RBD mutation pattern, that is, same signature mutations E484K and N501Y, except for K417 which is less impactful than E484 on antibody neutralization. This suggests possible involvement of other mutations, for instance, in NTD, which may contribute to enhanced resistance of beta VOC against neutralizing antibodies, and this gap of knowledge warrants further investigation.

The unprecedentedly dynamic and vast volume of SARS-CoV-2 genomic data from GISAID database has embodied the power of global and concerted effort on tracking the still-ongoing COVID-19 pandemic. The database is very informative and user-friendly, however, care must be taken when drawing conclusions based on the sequence data, and the following considerations infer the limitations of this study. First, as individual sequences were processed and submitted by various institutes worldwide, there might be variation in quality that we could hardly monitor. For instance, details on coverage and assembly method of some sequences were not provided by the submitters. Second, our data might not reflect the latest situation (especially for June and July 2021) due to highly variable lag time between collection and submission dates. Another factor to consider was inhomogeneous sampling. For instance, at continent level, sequences submitted by the USA comprised about $90 \%$ of North American sequences, and the situation of other countries might be underrepresented. At country level, we could not ensure submitted sequences were representative of the population without sampling bias towards particular lineages, nor for statistical data such as reported death tolls in certain countries. Another limitation was that we could not distinguish between locally transmitted and imported COVID-19 cases.

\section{Conclusions}

Our data showed that up till July 2021, delta VOC has predominated in 5 continents, and it appeared to be likely to replace gamma VOC in South America in near future. By monitoring prevalence of SARS-CoV-2 variants, we can track the emergence of 'the fittest and the fastest', and combination of variant prevalence and public health surveillance data might be useful for predicting next surge of COVID-19 cases. Surveillance of SARS-CoV-2 variants is particularly important for under-vaccinated regions with limited medical resources to minimize fatality. The breakthrough by delta VOCs in countries with higher vaccination rate suggested a broader immunization coverage to reach the threshold of herd immunity and vaccines with improved efficacy against major variants. 


\section{Abbreviations}

COVID-19: Coronavirus disease 2019; GISAID: Global Initiative on Sharing All Influenza Data; hACE2: Human angiotensin-converting enzyme 2; HR1 and 2: heptad repeats 1 and 2; NTD: N-terminal domain; RBD: Receptor-binding domain; S1 and S2: Subunits 1 and 2; SP: Signal peptide; SARS-CoV-2: Severe acute respiratory syndrome coronavirus 2; VOC: Variant of concern; VOI: Variant of interest; WHO: World Health Organization.

\section{Declarations}

\section{Ethics approval and consent to participate}

Not applicable.

\section{Consent for publication}

Not applicable.

\section{Availability of data and materials}

The datasets used and/or analyzed during the current study are available from the corresponding author on reasonable request.

\section{Competing interests}

The authors declare that they have no competing interests.

\section{Funding}

This research received no external funding.

\section{Authors' contributions}

BSFT and WSC conceptualized this study; WSC wrote the original draft; BSFT, ESKM, TLC, YML, JHYL and WSC analyzed the data and reviewed the manuscript. All authors read and approved the final manuscript.

\section{Acknowledgements}

We thank all institutions that shared their SARS-CoV-2 sequences to GISAID EpiCoV ${ }^{\text {TM }}$ database, which embodied 'sharing saves lives'.

\section{References}

1. Duffy S, Shackelton LA, Holmes EC. Rates of evolutionary change in viruses: patterns and determinants. Nat Rev Genet. 2008;9(4):267-76.

2. van Dorp L, Richard D, Tan CCS, Shaw LP, Acman M, Balloux F. No evidence for increased transmissibility from recurrent mutations in SARS-CoV-2. Nat Commun. 2020; 11(1):5986.

3. Korber B, Fischer WM, Gnanakaran S, Yoon H, Theiler J, Abfalterer W, et al. Tracking changes in SARS-CoV-2 spike: evidence that D614G increases infectivity of the COVID-19 virus. Cell. 2020;182(4):812-27.e19.

4. Leung K, Shum MH, Leung GM, Lam TT, Wu JT. Early transmissibility assessment of the N501Y mutant strains of SARS-CoV-2 in the United Kingdom, October to November 2020. Euro Surveill. 2021;26(1):2002106.

5. Tegally H, Wilkinson E, Lessells RJ, Giandhari J, Pillay S, Msomi N, et al. Sixteen novel lineages of SARS-CoV-2 in South Africa. Nat Med. 2021;27(3):440-6.

6. Huang Y, Yang C, Xu XF, Xu W, Liu SW. Structural and functional properties of SARS-CoV-2 spike protein: potential antivirus drug development for COVID-19. Acta Pharmacol Sin. 2020;41(9):1141-9.

7. Shang J, Wan Y, Luo C, Ye G, Geng Q, Auerbach A, et al. Cell entry mechanisms of SARS-CoV-2. Proc Natl Acad Sci U S A. 2020;117(21):11727-34.

8. World Health Organization. Tracking SARS-CoV-2 variants. https://www.who.int/en/activities/tracking-SARS-CoV-2-variants/. Accessed on 21 July 2021. 
9. Global Initiative on Sharing All Influenza Data (GISAID). EpiCoV ${ }^{\text {TM }}$ database. https://www.epicov.org/epi3/frontend\#63d1b2. Accessed on 16 July 2021.

10. Google News. Coronavirus (COVID-19). https://news.google.com/covid19/map?hl=en-

US\&mid=\%2Fm\%2F02j71\&gl=US\&ceid=US\%3Aen. Accessed on 16 July 2021.

11. World Health Organization. WHO Coronavirus (COVID-19) Dashboard. https://covid19.who.int. Accessed on 18 August 2021.

12. American Society for Microbiology. How dangerous is the delta variant (B.1.617.2)? https://asm.org/Articles/2021/July/HowDangerous-is-the-Delta-Variant-B-1-617-2. Accessed on 3 Aug 2021.

13. Ji Y, Ma Z, Peppelenbosch MP, Pan Q. Potential association between COVID-19 mortality and health-care resource availability. Lancet Glob Health. 2020;8(4):e480.

14. World Health Organization. WHO validates Sinovac COVID-19 vaccine for emergency use and issues interim policy recommendations. https://www.who.int/news/item/01-06-2021-who-validates-sinovac-covid-19-vaccine-for-emergency-use-andissues-interim-policy-recommendations. Accessed on 30 June 2021.

15. Goes LR, Siqueira JD, Garrido MM, Alves BM, Pereira ACPM, Cicala C, et al. New infections by SARS-CoV-2 var-iants of concern after natural infections and post-vaccination in Rio de Janeiro, Brazil. Infect Genet Evol. 2021;94:104998.

16. Estofolete CF, Banho CA, Campos GRF, Marques BC, Sacchetto L, Ullmann LS, et al. Case Study of Two Post Vac-cination SARS-CoV-2 Infections with P1 Variants in CoronaVac Vaccinees in Brazil. Viruses. 2021;13(7):1237.

17. Hacisuleyman E, Hale C, Saito Y, Blachere NE, Bergh M, Conlon EG, et al. Vaccine Breakthrough Infections with SARS-CoV-2 Variants. N Engl J Med. 2021;384(23):2212-8.

18. Williams SV, Vusirikala A, Ladhani SN, Fernandez Ruiz De Olano E, lyanger N, Aiano F, et al. An outbreak caused by the SARS-CoV-2 Delta (B.1.617.2) variant in a care home after partial vaccination with a single dose of the COVID-19 vaccine Vaxzevria, London, England, April 2021. Euro Surveill. 2021;26(27):2100626.

19. Lumley SF, Rodger G, Constantinides B, Sanderson N, Chau KK, Street TL, et al. An observational cohort study on the incidence of SARS-CoV-2 infection and B.1.1.7 variant infection in healthcare workers by antibody and vaccination status. Clin Infect Dis. 2021:ciab608.

20. Sheikh A, McMenamin J, Taylor B, Robertson C, Public Health Scotland and the EAVE II Collaborators. SARS-CoV-2 Delta VOC in Scotland: demographics, risk of hospital admission, and vaccine effectiveness. Lancet. 2021;397(10293):2461-2.

21. Amatya B, Pandey P, Dawadi S, Manandhar S. COVID-19 in fully vaccinated Everest trekkers in Nepal. J Travel Med. 2021:taab098.

22. Chen $Y$, Shen $H$, Huang R, Tong X, Wu C. Serum neutralising activity against SARS-CoV-2 variants elicited by CoronaVac. Lancet Infect Dis. 2021;21(8):1071-2.

23. Cao Y, Yisimayi A, Bai Y, Huang W, Li X, Zhang Z, et al. Humoral immune response to circulating SARS-CoV-2 variants elicited by inactivated and RBD-subunit vaccines. Cell Res. 2021;31(7):732-41.

24. Lustig Y, Zuckerman N, Nemet I, Atari N, Kliker L, Regev-Yochay G, et al. Neutralising capacity against Delta (B.1.617.2) and other variants of concern following Comirnaty (BNT162b2, BioNTech/Pfizer) vaccination in health care workers, Israel. Euro Surveill. 2021;26(26):2100557.

25. Madhi SA, Baillie V, Cutland CL, Voysey M, Koen AL, Fairlie L, et al. Efficacy of the ChAdOx1 nCoV-19 Covid-19 Vaccine against the B.1.351 Variant. N Engl J Med. 2021;384(20):1885-98.

26. Shen X, Tang H, Pajon R, Smith G, Glenn GM, Shi W, et al. Neutralization of SARS-CoV-2 Variants B.1.429 and B.1.351. N Engl J Med. 2021;384(24):2352-4.

27. Centers for Disease Control and Prevention. SARS-CoV-2 Variant Classifications and Definitions. https://www.cdc.gov/coronavirus/2019-ncov/cases-updates/variant-surveillance/variant-info.html. Accessed on 4 Aug 2021.

28. Barnes CO, West AP Jr, Huey-Tubman KE, Hoffmann MAG, Sharaf NG, Hoffman PR, et al. Structures of Human Antibodies Bound to SARS-CoV-2 Spike Reveal Common Epitopes and Recurrent Features of Antibodies. Cell. 2020;182(4):828-42.e16.

29. Valdes-Balbin Y, Santana-Mederos D, Paquet F, Fernandez S, Climent Y, Chiodo F, et al. Molecular Aspects Concerning the Use of the SARS-CoV-2 Receptor Binding Domain as a Target for Preventive Vaccines. ACS Cent Sci. 2021;7(5):757-67.

30. Greaney AJ, Starr TN, Gilchuk P, Zost SJ, Binshtein E, Loes AN, et al. Complete Mapping of Mutations to the SARS-CoV-2 Spike Receptor-Binding Domain that Escape Antibody Recognition. Cell Host Microbe. 2021;29(1):44-57.e9.

31. Weisblum Y, Schmidt F, Zhang F, DaSilva J, Poston D, Lorenzi JC, et al. Escape from neutralizing antibodies by SARS-CoV-2 spike protein variants. Elife. 2020;9:e61312. 
32. Baum A, Fulton BO, Wloga E, Copin R, Pascal KE, Russo V, et al. Antibody cocktail to SARS-CoV-2 spike protein prevents rapid mutational escape seen with individual antibodies. Science. 2020;369(6506):1014-8.

\section{Table}


Table 1

Spike protein mutations among SARS-CoV-2 VOCs and VOIs. The cells with ticks and bracketed ticks denote high and partial prevalence in the lineages, respectively. Variants with asterisks are former VOIs. HR1 and 2, heptad repeats 1 and 2; NTD, N-terminal domain; RBD, receptor-binding domain; S1 and S2, subunits 1 and 2; SP, signal peptide.

\begin{tabular}{|c|c|c|c|c|c|c|c|c|c|c|c|c|}
\hline \multirow[t]{2}{*}{ Mutations } & \multicolumn{4}{|l|}{ VOCs } & \multicolumn{7}{|l|}{ VOls } & \multirow{2}{*}{$\begin{array}{l}\text { Locations } \\
\text { in } \\
\text { spike } \\
\text { protein }\end{array}$} \\
\hline & Alpha & Beta & Gamma & Delta & Epsilon* & Zeta* & Eta & Theta* & lota & Kappa & Lambda & \\
\hline L5F & & & & & & & & & $\sqrt{ }$ & & & SP \\
\hline S13I & & & & & $\sqrt{ }$ & & & & & & & \\
\hline L18F & & & $\sqrt{ }$ & & & & & & & & & NTD \\
\hline T19R & & & & $\sqrt{ }$ & & & & & & & & \\
\hline $\mathrm{T} 20 \mathrm{~N}$ & & & $\sqrt{ }$ & & & & & & & & & \\
\hline P26S & & & $\sqrt{ }$ & & & & & & & & & \\
\hline A67V & & & & & & & $\sqrt{ }$ & & & & & \\
\hline 69/70del & $\sqrt{ }$ & & & & & & $\sqrt{ }$ & & & & & \\
\hline V70F & & & & $(\sqrt{ })$ & & & & & & & & \\
\hline G75V & & & & & & & & & & & $\sqrt{ }$ & \\
\hline T76I & & & & & & & & & & & $\sqrt{ }$ & \\
\hline D80A & & $\sqrt{ }$ & & & & & & & & & & \\
\hline D80G & & & & & & & & & $(\sqrt{ })$ & & & \\
\hline T95I & & & & $\sqrt{ }$ & & & & & $\sqrt{ }$ & $(\sqrt{ })$ & & \\
\hline D138Y & & & $\sqrt{ }$ & & & & & & & & & \\
\hline 141/143del & & & & & & & & $(\sqrt{ })$ & & & & \\
\hline G142D & & & & $\sqrt{ }$ & & & & & & $\sqrt{ }$ & & \\
\hline 144del & $\sqrt{ }$ & & & & & & $\sqrt{ }$ & & $(\sqrt{ })$ & & & \\
\hline W152C & & & & & $\sqrt{ }$ & & & & & & & \\
\hline E154K & & & & & & & & & & $\sqrt{ }$ & & \\
\hline 156/157del & & & & $\sqrt{ }$ & & & & & & & & \\
\hline F157S & & & & & & & & & $(\sqrt{ })$ & & & \\
\hline R158G & & & & $\sqrt{ }$ & & & & & & & & \\
\hline R190S & & & $\sqrt{ }$ & & & & & & & & & S1 \\
\hline D215G & & $\sqrt{ }$ & & & & & & & & & & \\
\hline A222V & & & & $(\sqrt{ })$ & & & & & & & & \\
\hline 241/242/243del & & $\sqrt{ }$ & & & & & & & & & & \\
\hline 243/244del & & & & & & & & $(\sqrt{ })$ & & & & \\
\hline 247/253del & & & & & & & & & & & $(\sqrt{ })$ & \\
\hline D253G & & & & & & & & & $\sqrt{ }$ & & & \\
\hline W258L & & & & $(\sqrt{ })$ & & & & & & & & \\
\hline Y265C & & & & & & & & $(\sqrt{ })$ & & & & \\
\hline K417N & & $\sqrt{ }$ & & $(\sqrt{ })$ & & & & & & & & RBD \\
\hline
\end{tabular}




\begin{tabular}{|c|c|c|c|c|c|c|c|c|c|c|c|c|}
\hline \multirow[t]{2}{*}{ Mutations } & \multicolumn{4}{|l|}{ Vocs } & \multicolumn{7}{|l|}{ VOls } & \multirow{2}{*}{$\begin{array}{l}\text { Locations } \\
\text { in } \\
\text { spike } \\
\text { protein }\end{array}$} \\
\hline & Alpha & Beta & Gamma & Delta & Epsilon* & Zeta* & Eta & Theta* & lota & Kappa & Lambda & \\
\hline K417T & & & $\sqrt{ }$ & & & & & & & & & \\
\hline L452Q & & & & & & & & & & & $\sqrt{ }$ & \\
\hline L452R & & & & $\sqrt{ }$ & $\sqrt{ }$ & & & & $(\sqrt{ })$ & $\sqrt{ }$ & & \\
\hline S477N & & & & & & & & & $(\sqrt{ })$ & & & \\
\hline T478K & & & & $\sqrt{ }$ & & & & & & & & \\
\hline E484K & $(\sqrt{ })$ & $\sqrt{ }$ & $\sqrt{ }$ & & & $\sqrt{ }$ & $\sqrt{ }$ & $(\sqrt{ })$ & $\sqrt{ }$ & & & \\
\hline E484Q & & & & & & & & & & $\sqrt{ }$ & & \\
\hline F490S & & & & & & & & & & & $\sqrt{ }$ & \\
\hline S494P & $(\sqrt{ })$ & & & & & & & & & & & \\
\hline N501Y & $\sqrt{ }$ & $\sqrt{ }$ & $\sqrt{ }$ & & & & & $(\sqrt{ })$ & & & & \\
\hline F565L & & & & & & $(\sqrt{ })$ & & & & & & \\
\hline A570D & $\sqrt{ }$ & & & & & & & & & & & \\
\hline D614G & $\sqrt{ }$ & $\sqrt{ }$ & $\sqrt{ }$ & $\sqrt{ }$ & $\sqrt{ }$ & $\sqrt{ }$ & $\sqrt{ }$ & $\sqrt{ }$ & $\sqrt{ }$ & $\sqrt{ }$ & $\sqrt{ }$ & \\
\hline H655Y & & & $\sqrt{ }$ & & & & & & & & & \\
\hline Q677H & & & & & & & $\sqrt{ }$ & & & & & \\
\hline P681H & $\sqrt{ }$ & & & & & & & $\sqrt{ }$ & & & & \\
\hline P681R & & & & $\sqrt{ }$ & & & & & & $\sqrt{ }$ & & \\
\hline A701V & & $\sqrt{ }$ & & & & & & & $\sqrt{ }$ & & & $\mathrm{S} 2$ \\
\hline T716I & $\sqrt{ }$ & & & & & & & & & & & \\
\hline T859N & & & & & & & & & $(\sqrt{ })$ & & $\sqrt{ }$ & \\
\hline F888L & & & & & & & $\sqrt{ }$ & & & & & \\
\hline D950N & & & & $\sqrt{ }$ & & & & & & & & HR1 \\
\hline $\mathrm{D} 950 \mathrm{H}$ & & & & & & & & & $(\sqrt{ })$ & & & \\
\hline Q957R & & & & & & & & & $(\sqrt{ })$ & & & \\
\hline S982A & $\sqrt{ }$ & & & & & & & & & & & \\
\hline T1027I & & & $\sqrt{ }$ & & & & & & & & & \\
\hline Q1071H & & & & & & & & & & $\sqrt{ }$ & & \\
\hline E1092K & & & & & & & & $\sqrt{ }$ & & & & \\
\hline H1101Y & & & & & & & & $\sqrt{ }$ & & & & \\
\hline D1118H & $\sqrt{ }$ & & & & & & & & & & & \\
\hline V1176F & & & & & & $\sqrt{ }$ & & $\sqrt{ }$ & & & & HR2 \\
\hline K1191N & $(\sqrt{ })$ & & & & & & & & & & & \\
\hline
\end{tabular}

\section{Figures}




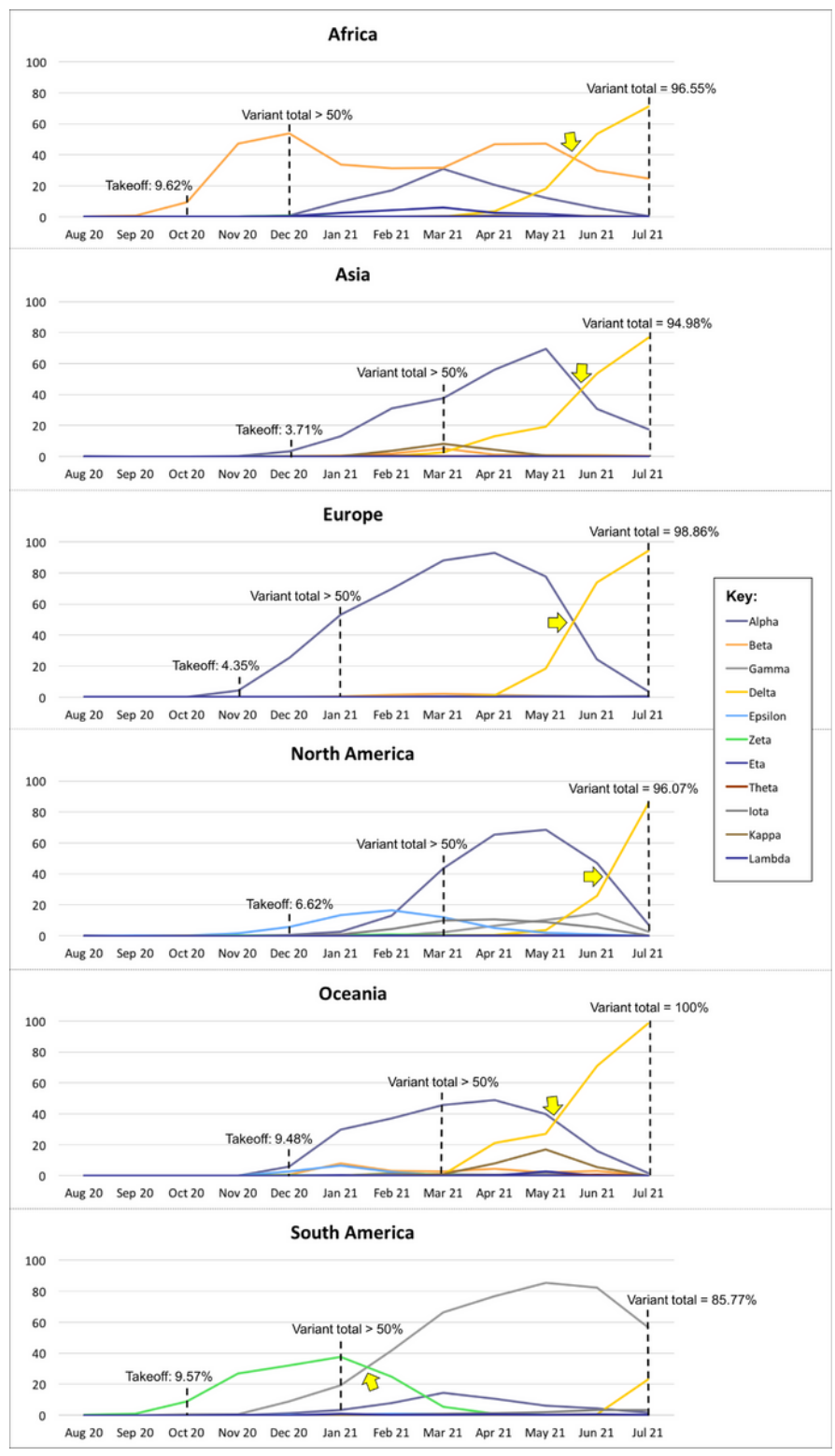

Figure 1

Proportions of SARS-CoV-2 variants collected in 6 continents throughout August 2020 to July 2021. The yellow arrows indicate the time points of major lineage interchange. 


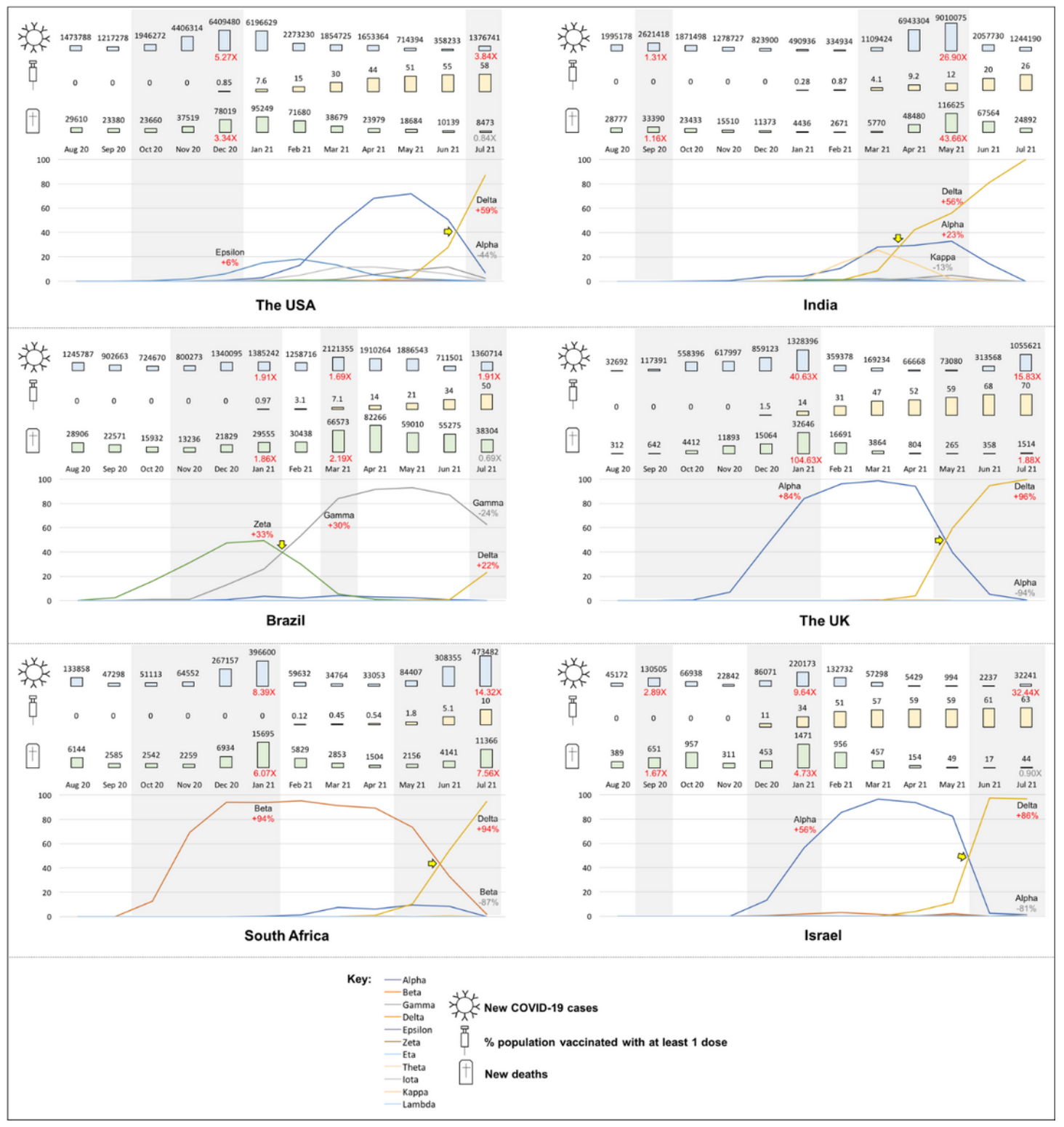

\section{Figure 2}

Monthly data on new COVID-19 cases and deaths, vaccination status and proportions of SARS-CoV-2 variants. Grey areas cover the ascending phases of new COVID-19 cases. Yellow arrows indicate the time points of major lineage interchange. The fold and percentage changes were relative to the baseline (the value right before the start of an ascending phase). 